\title{
Informed consent to microsatellite instability and immunohistochemistry screening for Lynch syndrome
}

\author{
Barbara Chubak, $M D^{I}$, Brandie Heald, $M S^{2}$, and Richard R. Sharp, PhD ${ }^{1,2,3}$
}

\begin{abstract}
Objective: Routine microsatellite instability and immunohistochemistry screening of colorectal cancers can assist in identifying a significant proportion of cancers attributable to Lynch syndrome. This article considers whether it is necessary to obtain patient informed consent for microsatellite instability and immunohistochemistry screening. Results: Although microsatellite instability screening examines genetic features of a tumor, it lacks several important characteristics that typically mandate formal informed consent to genetic testing. Microsatellite instability screening describes discrete tissue samples and does not provide information about the rest of the patient's body or germline. In contrast, immunohistochemistry screening is a proteomic test that may reveal information about the patient's germline. As such, immunohistochemistry screening can be viewed as similar to other forms of genetic testing, in which explicit patient consent is regarded as an ethical prerequisite. Conclusion: There is no ethical requirement to obtain explicit informed consent for microsatellite instability screening of colorectal tumor samples for Lynch syndrome. There is support for obtaining patient consent to immunohistochemistry testing, given its similarities with other genetic analyses for which informed consent is typically deemed necessary. Regardless of which screening test is used, it is important to prepare patients and their families for the possibility of a positive screening test. Genet Med 2011:13(4):356-360.
\end{abstract}

Key Words: informed consent, ethics, MSI screening, IHC screening, Lynch syndrome, HNPCC, colorectal cancer

$\mathrm{C}^{\mathrm{o}}$ olorectal cancer (CRC) is the third most common cancer in the United States and the third leading cause of cancer-related mortality in American men and women. ${ }^{1}$ Some CRCs arise in the setting of highly penetrant, dominant-inherited syndromes, including Lynch syndrome (LS), which accounts for 3-5\% of CRCs. ${ }^{2}$ It is important to identify patients with LS (also known as hereditary nonpolyposis colorectal cancer), because these individuals have an increased risk of developing a second primary CRC and cancer of the endometrium, ovaries, stomach, small bowel, bile ducts, pancreas, brain, and transitional cell carcinoma of the renal pelvis and ureter. ${ }^{3}$ In addition to receiving treatment for known cancers, standard care for patients with LS includes routine colonoscopic surveillance and sometimes prophylactic removal of the organs most often affected by the syndrome. ${ }^{4}$

LS is diagnosed using germline genetic testing to identify a causative mutation in one of four DNA mismatch repair (MMR)

From the ${ }^{1}$ Department of Bioethics, Cleveland Clinic; ${ }^{2}$ Genomic Medicine Institute Lerner College of Medicine, Cleveland Clinic; and ${ }^{3} \mathrm{Center}$ for Genomic Research Ethics \& Law, Case Western Reserve University, Cleveland, Ohio.

Richard R. Sharp, PhD, Department of Bioethics, JJ-60, Cleveland Clinic, 9500 Euclid Ave, Cleveland, OH 44195. E-mail: sharpr3@ccf.org.

Disclosure: The authors declare no conflict of interest.

Submitted for publication June 1, 2010.

Accepted for publication December 9, 2010

Published online ahead of print March 14, 2011

DOI: 10.1097/GIM.0b013e31820aee09 genes. ${ }^{5}$ However, genetic evaluation of these MMR genes is not recommended for all patients with $\mathrm{CRC}$, because for the majority of these patients, the risks of testing - including the expense entailed and the psychosocial risks to the patient that frequently accompany such testing - typically outweigh the benefits. ${ }^{2}$ For this reason, a considerable portion of the literature about LS and other inherited cancer syndromes is devoted to discussing the ethics of diagnosis. Sections on informed consent and the psychosocial hazards of test results feature prominently in many articles about genetic testing for CRC. . $^{2,6-8}$

The need for patient informed consent is an ethical and legal touchstone of contemporary medicine, and a prerequisite for both colonoscopic disease surveillance and genetic evaluation of MMR genes. However, there are two screening tests which are widely used for identifying a subset of patients with CRC who are at elevated risk of having LS for which the need for informed consent is unclear. These screening tests are microsatellite instability (MSI) testing and immunohistochemistry (IHC) of resected CRC tissue.

This article considers the need for explicit patient consent to MSI and IHC as screening tests to identify increased risk of LS. We argue that explicit patient informed consent is not an ethical requirement for MSI screening but may be appropriate in the case of IHC, a context in which explicit patient consent is not typically viewed as necessary. The position we advocate is supported by an ethical analysis of informed-consent expectations and departs substantially from the conclusions reached by a recent Centers for Disease Control and Prevention Working Group, ${ }^{9}$ which recommended that patient consent be obtained before MSI and IHC screening to assess LS risk.

\section{MSI SCREENING FOR LS}

LS lacks a distinctive clinical phenotype, and the pedigreebased Amsterdam Criteria have proven to correlate poorly with the syndrome. As a result, its diagnosis is now defined in genetic terms. ${ }^{10}$ Patients with LS have a germline mutation in one or more MMR genes-MLH1, MSH2, MSH6, or PMS2the abnormal expression of which is thought to facilitate cells' malignant transformation, by promoting the accumulation of mutations. The Bethesda Guidelines were developed and recently revised as an aid to determine which patients with CRC should undergo MSI screening. ${ }^{11}$ Unfortunately, large-scale studies have shown these guidelines to have poor sensitivity and specificity, leading to the recommendation that MSI screening be made available to all patients with CRC, regardless of whether they meet traditional criteria for LS. ${ }^{9}$ A recent study assessing cost-effectiveness confirmed the appropriateness of this recommendation. ${ }^{12}$

MSI is a genetic screening test, which uses variability in the lengths of microsatellite markers as a surrogate for defects in DNA MMR mechanisms. Microsatellites are stretches of DNA in which a short motif is repeated several times. In the normal course of DNA replication and cell division, microsatellites are replicated exactly, so that they are identical between cells. However, defective MMR can cause microsatellites to become 
unstable, gaining or losing repeats to vary in length between cells. MSI screening compares microsatellite lengths between patients' neoplastic tissue and normal tissue, which may be obtained from blood, a buccal smear, or most often, from normal colonic mucosa. ${ }^{13}$ Typically, a panel of five microsatellite markers is used, with instability in two or more signifying a positive or MSI-high (MSI-H) result. A clinically insignificant MSI-low result features instability in only one microsatellite, whereas a microsatellite-stable result has no differences in the lengths of the microsatellites tested. ${ }^{5}$

Not all patients with CRC have MSI, and not all patients who test MSI-H have LS; $10-15 \%$ of sporadic CRCs are MSI-H, as a result of acquired hypermethylation of the $M L H 1$ promoter. ${ }^{14}$ For this reason, MSI is not considered diagnostic of LS but suggestive of MMR mutation, with a sensitivity of $89 \%$ for MLH1 and MSH 2 mutations and $77 \%$ for MSH6 mutation. ${ }^{9}$ It is recommended that MSI-H test results be followed by germline testing of all four MMR genes, to make a diagnosis of LS. ${ }^{2}$ In addition to suggesting the presence of a mutation in one of the MMR genes, MSI is associated with insensitivity to fluorouracil adjuvant chemotherapy. ${ }^{15}$ Additional research is necessary to demonstrate whether and how this association should impact clinical care for patients who test MSI-H. ${ }^{9}$

\section{IHC SCREENING FOR LS}

An alternative to MSI screening is the use of IHC to screen patients who may have MMR mutations. IHC dates back to the 1940s and is a well-established part of clinical medicine's armamentarium, often used by pathologists to describe and diagnose disease. This screening modality uses chemical markers to determine the presence or absence of gene products. In the case of LS, the products of interest are those from MMR genes that are associated with the syndrome. MMR proteins are only stable in heterodimer pairs of "major" and "minor" proteins, so most IHC is conducted using antibodies against the more informative "major" MLH1 and MSH2 proteins, but antibodies against all four MMR proteins may be used. ${ }^{12}$

When IHC staining suggests that one or more MMR proteins are absent from a patient's tumor tissue, that patient is considered to have abnormal IHC. The sensitivity of IHC as an indication of MMR mutation is $83 \%$, and the specificity is $90 \%$. Similar to MSI screening, IHC cannot distinguish between lack of expression due to germline mutation and epigenetic silencing from hypermethylation. In this regard, both MSI screening and IHC are suggestive of heritable MMR mutation but are not considered diagnostic. ${ }^{5}$ In contrast to MSI screening, however, a positive IHC test suggests that not only one of the MMR genes is mutated but also which MMR gene is implicated. ${ }^{16}$

The apparent inability of the Amsterdam Criteria and Bethesda Guidelines to identify a significant minority of LS cases suggests that IHC, similar to MSI, is best applied to all cases of CRC. This is feasible because as a longstanding fixture of clinical pathology, IHC is currently more widely available and less expensive than MSI screening, which requires tumor microdissection and access to a specialized molecular diagnostics laboratory. ${ }^{17}$ As IHC can detect which of the MMR proteins tested is absent, it can also guide the search for germline mutations that may follow toward a specific MMR gene, potentially increasing efficiency and reducing the cost of subsequent diagnostic genetic testing. ${ }^{16}$

Both MSI and IHC have their respective benefits and limitations. As a result, some physicians use both when evaluating CRC tissue. ${ }^{7}$ Whether one modality should be preferred is a source of ongoing debate. ${ }^{16}$

\section{EXPECTATIONS OF INFORMED CONSENT}

Expectations about informed consent, in which a patient is informed of the risks, benefits, and alternatives to a procedure before it is performed, reflect a combination of ethical and legal perspectives. ${ }^{18}$ Foundational to informed consent is the bioethical principle of patient autonomy, which grants patients who are capable of self-rule sovereignty over their bodies, with understanding and control of what is done to them. In theory, the informed-consent process assures and confirms patient autonomy for all medical interventions. In practice, specific expectations regarding the need to obtain informed consent can have less to do with self-determination and more to do with the law. Decades of professional experience and legal cases have shaped the application of informed consent, so that its precision and explicitness vary with circumstance and the perceived level of risk. ${ }^{19}$ For example, a blood test for HIV, which is understood to carry significant psychosocial risks, must be preceded by the patient's explicit informed consent. In contrast, for less risky diagnostic tests, the patient's informal agreement, gesturally implied by his/her cooperation with phlebotomy, is often viewed as sufficient.

In the case of CRC, it is generally expected that a patient or his/her legally authorized surrogate must give explicit informed consent to colonoscopy or surgery. This expectation reflects the level of personal invasiveness and risk associated with these interventions. Unlike implied forms of patient agreement, explicit consent involves the direct solicitation of a patient's willingness to proceed with a specific procedure, diagnostic test, or treatment. Before seeking explicit consent, clinicians are expected to explain and assess the patient's understanding of any associated risks, potential benefits, and medical alternatives. Often, the added step is taken of documenting this informed consent with a patient's signature. However, no comparable expectation exists for the subsequent pathologic processing of any biopsied or resected tissue. ${ }^{20}$ For these postoperative procedures, much like a low-risk blood test, consent practices typically are informal and implicit, with the patient's consent to undergo surgery viewed as encompassing subsequent diagnostic evaluation of resected tissue. The specific threshold of invasiveness and risk to the patient that must be reached for a medical intervention to require explicit, rather than implicit, informed consent is constantly in flux, dependent on current psychosocial concerns, medical practices, and legal developments.

Genetic tests are considered among those medical interventions that generally require a patient's explicit informed consent. Physically, these tests are minimally invasive and low risk, conducted on biopsied tissue, a buccal smear, or blood. However, their biocultural status is more complex, with genetic tests having potential medical and social implications, not only for the individual patient but also for his/her family and the larger biological community of which the patient is a member. ${ }^{21} \mathrm{Of}$ particular concern is a tendency for many patients to read too much into genetic data and to perceive probability as fate. This understanding of genetic test results can "make well people sick," tainting healthy patients' perception of their bodies and those of their family. ${ }^{6}$ Also, despite the recent passage of the Genetic Information Nondiscrimination Act, concerns remain that genetic test results might be used to deny employment or increase health and life insurance costs for patients and their family members who are identified as having genetic predispositions to disease. ${ }^{22}$ These considerations illustrate why genetic tests not only require explicit patient consent but also have engendered expectations that testing be preceded by formal genetic counseling. ${ }^{23}$ 
The importance of genetic counseling and explicit informed consent to genetic testing is underscored by statements such as those made by the American Society of Clinical Oncology, which recently reaffirmed its policy that, "Genetic testing only be done in the setting of pre- and post-test counseling, which should include discussion of possible risks and benefits of cancer early detection and prevention modalities." 24 In contrast, many nongenetic tests that are performed using tissues that have been removed from the patient are not viewed as reaching a threshold of risk that obligates formal patient consent. As such, medical practice typically involves the conduct of these nongenetic tests following less involved and largely implicit forms of patient agreement.

\section{SHOULD INFORMED CONSENT BE SOUGHT BEFORE CRC SCREENING?}

MSI screening is a genetic test and early cancer detection and prevention modality of the type discussed by the American Society of Clinical Oncology. It is performed for the sole purpose of assessing whether a patient is likely to have LS, with its risk of additional cancers in the future. It involves the direct analysis of DNA and is consistent with the definition of genetic testing as "an analysis of human DNA, RNA, chromosomes, proteins, or metabolites that detects genotypes, mutations, or chromosomal changes." 25 As a genetic test, MSI might be regarded as requiring explicit patient consent. This is the conclusion reached by the Centers for Disease Control and Prevention panel on the Evaluation of Genomic Applications in Practice and Prevention (EGAPP) Working Group, which has recommended "With limited benefit of genetic testing to the CRC patient, the EWG recommends that informed consent should be obtained before MSI or IHC testing." 9

The EGAPP Working Group notes that there is "general debate on the issue of consent" to MSI screening, ${ }^{9}$ but there is little evidence of this debate within the published literature. Debates about MSI screening for LS have neither elucidated clear points of ethical consensus nor have they given rise to practice guidelines on the need to obtain explicit patient consent. Within departments of oncology and genetic medicine, and at conferences of genetic counselors and colorectal surgeons, debate about the need for patient informed consent continues. ${ }^{26}$ Part of the reason for this debate is pragmatic: if informed consent to MSI is to be obtained, as the EGAPP Working Group suggests, when should it be obtained and who should be responsible? What information should the consent discussion include? If informed consent to MSI testing is indeed an ethical necessity, then it must be obtained, regardless of any inconvenience associated with the process.

Contrary to the position of the EGAPP Working Group, we suggest that the rationale for obtaining explicit informed consent is not compelling in the case of MSI screening. MSI screening does not affect patients in any of the potentially harmful ways that typically make it important to obtain explicit patient consent to genetic testing. Many patients who are tested for MSI are already unwell, diagnosed with CRC, and surgically treated for it, substantially lessening any possibility of the psychosocial harm associated with making a well person sick. Unlike traditional forms of genetic testing, MSI screening does not describe the patient's body or the inherited properties of the patient's relatives. MSI analysis characterizes the resected CRC, not the patient's germline. Performed on tissue that has been resected for therapeutic purposes and describing only that tissue, MSI screening is similar in many ways to estrogen
Table 1 Factors relevant to an ethical requirement for specific informed consent to evaluation of inherited Lynch syndrome risk

\begin{tabular}{llll}
\hline Test characteristic & $\begin{array}{c}\text { Genetic } \\
\text { testing }\end{array}$ & $\begin{array}{c}\text { MSI } \\
\text { testing }\end{array}$ & $\begin{array}{c}\text { IHC } \\
\text { testing }\end{array}$ \\
\hline $\begin{array}{l}\text { Physically invasive or burdensome } \\
\text { on the proband }\end{array}$ & No & No & No \\
$\begin{array}{l}\text { Significant implications for patient } \\
\text { management (including medical } \\
\text { monitoring) }\end{array}$ & Yes & No & No \\
$\begin{array}{l}\text { Potential to redefine a patient's } \\
\text { view of themselves and their } \\
\text { health status }\end{array}$ & Yes & No & Yes \\
$\begin{array}{l}\text { Potential for psychosocial harm } \\
\text { (anxiety, stigmatization, } \\
\text { discrimination, etc.) }\end{array}$ & Low & Very low & Very low \\
$\begin{array}{l}\text { Can create moral obligations to } \\
\text { share results with the proband's } \\
\text { family }\end{array}$ & Yes & No & Unclear \\
\hline
\end{tabular}

receptor tumor typing for breast cancer and other nongenetic tests for which the prevailing medical practice is not to obtain explicit informed consent. Thus, the character and associated risk profile of MSI screening differ markedly from most genetic tests, in ways that argue against there being an ethical requirement of explicit informed consent to MSI screening for LS (Table 1).

Unlike MSI testing, IHC is generally not viewed as needing explicit patient consent. This may reflect a belief that IHC testing does not exceed the threshold of invasiveness and risk that is sufficient to trigger a requirement to obtain explicit patient consent. As a nongenetic test, routinely done without patient knowledge or agreement, the use of IHC as a screening tool for LS does not seem to have stimulated the same debate about consent as MSI screening. This is ironic, because unlike MSI screening, which characterizes the resected tumor without providing genetic information about the patient from whom it came, IHC can provide genetic information about the patient and, by extension, his/her family. Indeed, genetic findings from IHC screening can be quite specific. For example, IHC screening is able to pinpoint which MMR protein is absent and, therefore, which specific MMR gene is altered. Sporadic CRC frequently features loss of the MLH1 protein because of epigenetic changes, but loss of any of the other MMR proteins is generally indicative of a germline mutation of the gene for the absent protein. ${ }^{17}$

Because IHC screening of resected CRC tissue is able to indicate the presence of an inherited mutation in a specific gene, IHC has more in common with most genetic tests than MSI screening, including several characteristics which increase psychosocial risk and often generate an expectation of explicit patient consent (Table 1). For example, the results of IHC screening can be extrapolated from the patient to his/her family and can be interpreted in ways that make well people anticipate illness. Insofar as IHC can provide genetic information about the patient, and not simply about his/her resected CRC, it may be viewed as reaching a threshold of risk that necessitates explicit patient consent.

Nonetheless, the results of IHC are not considered diagnostic of LS, and the suggestion that formal informed consent be 
obtained before IHC screening represents a significant change in longstanding medical practice. For these reasons, further study to characterize the level of perceived risk (or actual harm) associated with IHC screening and to better assess the riskbenefit ratio of IHC screening is appropriate before a recommendation of explicit consent can be supported.

\section{RECOMMENDATIONS FOR PRACTICE}

Ultimately, both MSI-H and positive IHC screens must be followed by DNA sequencing to determine whether germline MMR mutations are present, if LS is to be diagnosed with certainty. $^{2}$ Unlike either MSI or IHC screening, DNA sequencing directly reveals information about the patient's heritable mutations and presents psychosocial risks that are typically associated with genetic testing. For this reason, it is essential that MMR gene sequencing be preceded by genetic counseling and explicit informed consent.

Although explicit informed consent is not an ethical prerequisite to MSI screening, and currently is not done routinely for IHC screening, it is appropriate to prepare patients for the possibility of a positive tumor screen and the accompanying recommendation that MMR gene sequencing be considered. This goal could be promoted by informing patients at the time of their diagnosis or treatment that CRC risk runs in families and that they will be contacted by a genetic counselor if their tumor is of a heritable type, to discuss the nature of their cancer risk further. Such a preparatory conversation would not only benefit the minority of patients who screen positively, priming them for future contact with a genetic counselor, but also those patients whose screens are negative, as these patients are likely to be unaware of the increased risk of CRC that is conferred on their family by virtue of their sporadic CRC. ${ }^{27}$

One opportunity for patient education is provided in the packet of informational documents that patients often are given to review preoperatively, which can be kept and reviewed by the patient or his/her family as needed. Another opportunity to educate patients about CRC risks is when the physician reports pathologic findings, including MSI and IHC test results. The patient might then be told, if his/her tumor has screened negative, that his/her family members have a slightly elevated risk of developing CRC compared with the average population and that enhanced colonoscopic screening measures are recommended. ${ }^{28}$ If his/her tumor has screened positive, the patient could then be advised that his/her tumor may be of a type that is inherited genetically and referral to a genetic counselor arranged to elaborate on the risks and benefits of the MMR testing that is required to confirm or reject the presence of LS. To increase the likelihood of patient follow-up, this referral to a genetic specialist might be coordinated, so as to coincide with the initial presentation of MSI and IHC test results.

\section{CONCLUSION}

In this article, we have discussed whether explicit patient informed consent is necessary to screen resected CRC for MSI or IHC consistent with LS. Professional debate on this topic has been characterized by unsupported assertions of consent requirements, with a tendency to assume that all genetic testing predictive of pathology requires explicit informed consent and comparatively little analysis of ethical arguments supporting this requirement. ${ }^{9}$ Our analysis of both the biology of MSI and IHC screening and the ethical foundations of an informedconsent requirement suggests that informed consent should not be viewed as a prerequisite for MSI screening, which charac- terizes a known CRC without describing the germline genetics of the postoperative patient.

In contrast, IHC for MMR proteins often does provide data about the patient's germline and, thus, may present some of the same psychosocial risks that frequently create expectations that patient consent be obtained. Because IHC is routinely performed in other contexts without patient consent, and because IHC screening is considered nondiagnostic of LS, it is unclear whether IHC reaches the threshold of risk that is typical of other genetic tests and that is sufficient to justify a requirement that explicit informed consent be obtained. To clarify this point, we recommend further empirical study to assess whether patients and health care providers would perceive a requirement of informed consent to IHC screening as appropriate in light of the potential benefits and risks involved. In addition, because the prevailing medical approach is to conduct IHC screening of patient tissues without informed consent, future research should seek to characterize best practices for seeking patient consent to IHC testing, along with the potential impact of a consent requirement on patient management. What remains uncontroversial, however, is that patients should be told about both positive and negative results of MSI and IHC screening to increase their understanding of familial CRC risk and to pave the way for future interactions with doctors, genetic counselors, and family members.

\section{REFERENCES}

1. American Cancer Society. Colorectal Cancer Facts and Figures 2008-2010. Available at: http://www.cancer.org/downloads/STT/F861708_finalforweb.pdf. Accessed April 27, 2010.

2. Burt R, Neklaston DW. Genetic testing for inherited colon cancer. Gastroenterology 2005;128:1696-1716.

3. Lynch HT, Smyrk TC, Watson P, et al. Genetics, natural history, tumor spectrum, and pathology of hereditary nonpolyposis colorectal cancer: an updated review. Gastroenterology 1993;104:1535-1549.

4. Vasen HF, Moslein G, Alonzo A, et al. Guidelines for the clinical management of Lynch syndrome (HNPCC). J Med Genet 2007;44:353-362.

5. Hampel H. Genetic testing for hereditary colorectal cancer. Surg Oncol Clin N Am 2009;18:687-703.

6. Chapman PD, Burn J. Genetic predictive testing for bowel cancer predisposition: the impact on the individual. Cytogenet Cell Genet 1999;86:118124

7. Kaz AM, Brentnall TA. Genetic testing for colon cancer. Nat Clin Pract 2006;3:670-679

8. Jarvinen HJ. Genetic testing for polyposis: practical and ethical aspects. Gut 2003;52(suppl 2):19-22.

9. EGAPP Working Group. Recommendations from the EGAPP Working Group: genetic testing strategies in newly diagnosed individuals with colorectal cancer aimed at reducing morbidity and mortality from Lynch syndrome in relatives. Genet Med 2009;11:35-41.

10. Kievit W, de Bruin JH, Adang EM, et al. Current clinical selection strategies for identification of hereditary non-polyposis colorectal cancer families are inadequate: a meta-analysis. Clin Genet 2004;126:42-48.

11. Umar A, Boland CR, Terdiman JP, et al. Revised Bethesda guidelines for hereditary non-polyposis colorectal cancer (Lynch syndrome) and microsatellite instability. J Natl Cancer Inst 2004;96:261-268.

12. Mvundura M, Grosse SD, Hampel H, et al. The cost-effectiveness of genetic testing strategies for Lynch syndrome among newly diagnosed patients with colorectal cancer. Genet Med 2010;10:1-12.

13. De la Chapelle A. Microsatellite instability. N Engl J Med 2003;349:209210 .

14. Lynch HT, Lynch PM, Lanspa SJ, et al. Review of the Lynch syndrome: history, molecular genetics, screening, differential diagnosis, and medicolegal ramifications. Clin Genet 2009;76:1-18

15. Ribic CM, Sargent DJ, Moore MJ, et al. Tumor microsatellite instability status as a predictor of benefit from fluorouracil-based adjuvant chemotherapy for colon cancer. $N$ Engl J Med 2003;349:247-257.

16. Shia J, Zhang L. Point/counterpoint: immunohistochemistry versus microsatellite instability testing for screening colorectal cancer patients at risk for hereditary nonpolyposis colon cancer syndrome. J Mol Diagn 2008;10:293307.

17. Hampel H, Frankel WL, Martin E, et al. Feasibility of screening for Lynch syndrome among patients with colorectal cancer. J Clin Oncol 2008;26: $5783-5788$. 
18. Faden R, Beauchamp T. A history and theory of informed consent. Oxford: Oxford University Press, 1986.

19. Berg JW, Appelbaum PS, Parker LS, et al. Informed consent: legal theory and clinical practice, 2nd ed. Oxford: Oxford University Press, 2001.

20. Feld AD. Medicolegal implications of colon cancer screening. Gastrointest Endosc Clin N Am 2002;12:171-179.

21. Juengst ET. FACE facts: why human genetics will always provoke bioethics. J Law Med Ethics 2004;32:267-275.

22. Rothstein M, Anderlik MA. What is genetic discrimination and when and how can it be prevented? Genet Med 2001;3:354-358.

23. Sorenson JR. Genetic counseling: values that have mattered. In: Bartels D, LeRoy B, Caplan A, editors. Prescribing our future: ethical challenges in genetic counseling. New York: Aldine de Gruyter, 1993:3-14.
24. American Society for Clinical Oncology (ASCO). ASCO policy statement update: genetic testing for cancer susceptibility. J Clin Oncol 2003;21:23972406.

25. Genetic Information Nondiscrimination Act (GINA). Pub. L. No. 110-233, 122 Stat. 881, 2008.

26. Sharp RR. Is informed consent necessary for MSI/IHC screening for HNPCC? Paper presented at American College of Medical Genetics. Albuquerque, NM, March 24, 2010.

27. Rubin DT, Gandhi RK, Hetzel JT, et al. Do colorectal cancer patients understand that their family is at risk? Dig Dis Sci 2009;54:2473-2483.

28. Fuchs CS, Giovannucci EL, Colditz GA, et al. A prospective study of family history and the risk of colorectal cancer. N Engl J Med 1994;331:16691674. 